\title{
Human African Trypanosomiasis in the Northern Region of Zimbabwe: Reported Cases, Health Implications and the Way Forward
}

Amon Munyenyiwa ${ }^{1,2,3 *}$, Moses Zimba ${ }^{3}$, Mascelini Jenipher Mutsaka-Makuvaza ${ }^{1}$, Portia Manangazira ${ }^{5}$, Tamuka Nhiwatiwa ${ }^{2,3}$, Charles Maponga ${ }^{4}$ and Nicholas Midzi ${ }^{1}$

${ }^{1}$ National Institute of Health Research, Zimbabwe

${ }^{2}$ University of Zimbabwe Lake Kariba Research Station, Zimbabwe

${ }^{3}$ Department of Biological Sciences, University of Zimbabwe, Mt. Pleasant, Harare, Zimbabwe

${ }^{4}$ Department of Pharmacy, University of Zimbabwe, Mt. Pleasant, Harare, Zimbabwe

${ }^{5}$ Epidemiology and Disease Control Department, Ministry of Health and Child Care, Harare, Zimbabwe

*Corresponding author: Amon Munyenyiwa, National Institute of Health Research, Zimbabwe; University of Zimbabwe Lake Kariba Research Station; Department of Biological Sciences, University of Zimbabwe, Mt. Pleasant, Harare, Zimbabwe; E-mail: amon.munyenyiwa92@gmail.com

\begin{abstract}
Human African Trypanosomiasis (HAT) keeps resurfacing in Zimbabwe raising the need to capacitate the health system with adequate tools to eliminate the disease as a public health threat in the country. The aim of this study is to document recorded HAT cases in Zimbabwe as well as highlighting the impact of the disease and potential control strategies. Published research articles with main focus on HAT in Africa and Zimbabwe were used. The country recorded zero cases of HAT from 1998 to 2004, contrary to that 28 cases and 3 deaths were recorded between 2005 and 2015 and the highest number of cases were recorded in 2012 (9 cases). The cases are restricted to the Zambezi Valley, mainly Hurungwe and Kariba Districts. The distribution of the disease is linked to the distribution of tsetse flies in the country and the current population at high risk are game rangers and tourists as most of the cases are being reported in game parks (for example Mana pools). This may affect the tourism sector negatively if control measures are not placed in time. Furthermore, limited diagnostic capacity and lack of health personnel that are specifically trained for HAT resulted in misdiagnosis of the disease in the country there by distorting the true epidemiological situation of the disease in the country. Hence there is need for a strengthened diagnostic and surveillance system that monitors both HAT and Animal African Trypanosomiasis (One Health Approach) if elimination is to be achieved by 2030.
\end{abstract}

\section{Keywords}

Human African Trypanosomiasis, Epidemiology, Cases, Diagnosis and clinical management, One Health

\section{Abbreviations}

HAT: Human African Trypanosomiasis; CATT: CardAgglutination Trypanosomiasis Test; AAT: Animal African Trypanosomiasis; WHO: World Health Organization; LAMP: Loop-Mediated Isothermal Amplification; CSF: Cerebrospinal Fluid

\section{Introduction}

Human African Trypanosomiasis (HAT) is a disease that affects populations in rural Africa, where the tsetse fly vector that transmits the causative trypanosome parasites thrives. There are two forms of HAT: One, known as gambiense HAT, which is endemic in West and Central Africa (24 countries) and accounts for over 95\% of current cases; the other, known as rhodesiense HAT, is endemic in East and southern Africa (13 countries) and accounts for the remainder of cases [1]. The etiologic agent for the disease in Zimbabwe is Trypanosoma brucei rhodesiense that is transmitted by tsetse flies of the genus Glossina [2]. Two species, $G$. 
morsitans morsitans and G. pallidipes are HAT vectors in Zimbabwe [2]. Human beings are the main reservoir of $T$. b. gambiense, while domestic and wild animals constitute the reservoir for $T . b$. rhodesiense [1].

In the $20^{\text {th }}$ century, HAT has killed millions of people globally and Zimbabwe is among the countries that were affected [3]. There is limited information on the current situation of the disease in the country. The disease has long been rejected in the National health surveillance system. Most of the studies in the country are focusing on AAT than HAT. Hence this article aims to document reported HAT cases in Zimbabwe, health complications associated with the disease and provide recommendations that will be used as a tool to eliminate the disease.

\section{Clinical Presentation, Diagnosis and Manage- ment}

Following the bite of an infected fly (both male and female can transmit infection), Trypanosoma brucei ssp multiply in the skin, usually forming a chancre. The trypanosome parasite spread from the skin in blood and lymphatics; characterized by high fever, headache malaise, and lymphadenopathy and in some individuals a rash [4]. Late manifestations include multi-organ damage, with disturbance of the liver, kidney and hormonal gland functions. The presence of parasites in the brain leads to intense headaches, delusions, tremor and eventually drowsiness, lethargy coma and death if not treated in time [4]. Changes to sleep-wake patterns are among the symptoms that characterize the disease, leading to the common name "sleeping sickness". Unlike gambiense HAT, rhodesiense HAT that is found in Zimbabwe is acute, and patients succumb within just a few weeks after infection.

Diagnosis of the disease or presence of diagnostic equipment is really a challenge in countries with a weak health system like Zimbabwe. In Africa, for any new diagnostic test of the disease to be applicable in the remote affected areas it needs to be quick, easy to undertake, inexpensive, and reliable [5]. Detecting HAT in patients is important in view of the deadly outcomes of the disease and the toxicity of the drugs used for its treatment [6]. Card-Agglutination Trypanosomiasis Test (CATT) is the widespread method used for the diagnosis of the disease though the method has its limitations that include limited sensitivity and high frequency of equivocal results [7]. The Polymerase Chain reaction is currently used to improve accuracy in Zimbabwe but its costly and not applicable in the field. Supplementary tools were developed recently but to dater they have only been introduced to a limited extent into routine use (for example the loop-mediated isothermal amplification (LAMP) [8,9]; and the immune trypanolysis test) [10]. The tools are expected to make a significant role in the elimination of the disease. However, the question on how such tests will be used in the future and in what settings remains unanswered.

Human African Trypanosomiasis poses problems for treatment as the drugs that are most effective are toxic. Different treatments are available against parasites present in the haemo-lymphatic system (first-stage) and those that have entered the brain (second-stage). Currently, lumbar puncture is required to select the appropriate drug. Unlike HAT gambiense that received attention recently and a new drug (Fexinidazole) has been developed and is used for the treatment of the first stage, Rhodesian HAT is still being managed by old drugs [1]. Currently Rhodesian HAT is the most neglected disease, still treated with an ancient drug (suramin) from 1920 and the awful i.v. melarsoprol in propylene glycol for the late stage [11]. Patients are usually very ill and so administration of these drugs requires care and skill, particularly where there is evidence of central nervous system involvement. Initial therapy should include measures to improve the patients' general health and nutritional status so that aggressive anti-trypansomal therapy can be given. Relapses may occur during a course of treatment, and acceptable criteria of cure must be achieved. These criteria include the absence of parasites in the peripheral blood and in cerebrospinal fluid (CSF), return to normal biochemical and cytological CSF values and physical and mental recovery of the patient. These should ideally continue to be monitored for 3 years following completion of treatment.

\section{Materials and Methods}

The literature review was conducted using the online databases PubMed and HINARI. The information used includes published reports from WHO and published articles, with particular emphasis on the epidemiology and Control of HAT in Zimbabwe. A systematic search strategy was employed using the following search terms: 'Africa', Zimbabwe and 'Human African Trypanosomiasis' (e.g., 'Trypanosoma brucei rhodesiense.'). The search was refined to manuscripts that reported the occurrence of AAT or HAT in Southern, Central, Eastern, and Northern Africa and the whole world.

\section{Results and Discussion}

In Zimbabwe, r-HAT is limited to one area in the Zambezi Valley [2]. The review of HAT cases in Zimbabwe was necessitated by the sudden increase in number of new cases in 2012 (9 cases) [12]. During the aforementioned year, Zimbabwe Parks and Wildlife Management Authority wrote to the directorate Epidemiology and Disease Control, Ministry of Health and Child Care, informing them about the cases of HAT, which kept resurfacing in the Zambezi valley (Mana pools). The country reported zero cases of HAT from 1998 to 2004 and contrary to that, 28 cases and 3 deaths 


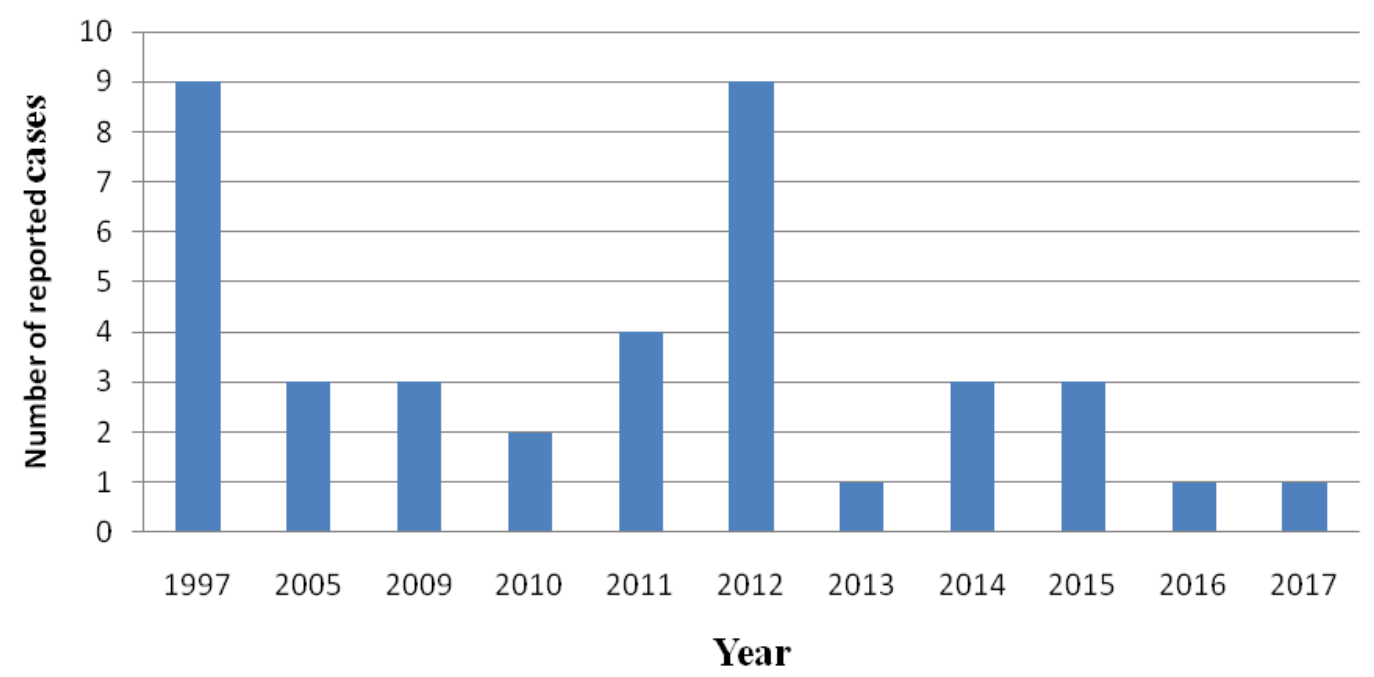

Figure 1: Trends of Human African Trypanosomiasis in Zimbabwe from 1997 to 2017 [12,13].

Note that the trends were based on the reported or recorded data available.

were recorded between 2005 and 2015 [2]. There have been at least 2 cases per year except in 2012, when 9 cases were reported for unknown reasons (Figure 1).

Possible hypotheses suggested wildlife declines or temperature changes in the area! In 2016 and 2017 the country reported each case per year. Historically, cases have been associated with proximity to the Zambezi River and wildlife, making the interface between wildlife and humans a major concern for r-HAT risk. This is especially important as the area is used for safari hunting groups and national parks visitors. Tsetse flies are present in an area of $20,000 \mathrm{~km}^{2}$ in the northern part of the country, and studies of their distribution through transects showed that sleeping sickness is linked to the distribution of tsetse-fly-vectors [2]. Many, HAT infections in the country were detected in game park rangers who spend most of their time in tsetse fly inhabitants as well as tourists who visited the Zambezi Valley (Hurungwe) [2].

Generally, there is still poor diagnostic capacity in health-care facilities, especially in the affected areas of Zimbabwe. Tools for HAT diagnosis and treatment are not readily available in the country and the cases are handled at St Anne's Hospital a private facility in Harare that is very expensive and unaffordable by those at high risk. Health-care workers are not trained in r-HAT specifically, which may be leading to misdiagnosis [12]. Furthermore, there is no treatment facility for r-HAT in the focus area, and cases are treated in Zambia or in hospitals in the capital city of Zimbabwe. However, there are plans to develop capacity in two hospitals that are close to the endemic area. Although training staff in smaller health facilities nearer to endemic areas could improve the power of detection; hospitals are always practically a better place to start. Currently, medicines are only dispensed in one pharmacy in Harare (which is over $200 \mathrm{~km}$ from the endemic areas).
There is a well-established tsetse control division under the Ministry of Lands, Agriculture, Fisheries, Water \& Rural Resettlement; department of Veterinary Sciences that is involved in eradicating the vector through various methods. The Ministry of Health and Child Care is responsible for the diagnosis and treatment of r-HAT, usually based on passive surveillance. In one active screening of 120 national parks staff, they found no cases. The National Neglected Tropical Diseases master plan has designated HAT as one of eight priorities [12]. Vector control is a key element of future activities, as the highest risk of infection occurs at the interface of protected areas and settled areas. The introduction of tsetse barriers at strategic locations may help prevent the dispersion of the vector into settled areas. A One Health approach supported by veterinary services has led to case management guidelines for rabies and anthrax, with plans to do the same for r-HAT, focusing on surveillance, rapid response, and improved case detection and reporting.

\section{Conclusion and Recommendations}

Limited diagnostic capacity of the disease in local health facilities, results in important levels of under detection and misdiagnosis thus distorting the true epidemiological situation of the disease in the country. A strengthened capacity for human sleeping sickness detection would enable a more realistic estimation of the burden of the disease in the country. Equipping health workers in endemic areas with the knowledge about the disease is critical if elimination has to be achieved by 2030 .

\section{Acknowledgements}

I would like to thank the Almighty for giving me the time to write this article in good health.

\section{Funding}

Authors received no specific funding for this work. 


\section{References}

1. World Health Organization (2021) Trypanosomiasis, human African (sleeping sickness).

2. Shereni W, Anderson NE, Nyakupinda L, Cecchi G (2016) Spatial distribution and trypanosome infection of tsetse flies in the sleeping sickness focus of Zimbabwe in Hurungwe District. Parasites \& Vectors 9: 605.

3. Mulenga P, Boelaert M, Lutumba P, Kelen VC, Coppieters $Y$, et al. (2019) Integration of Human African Trypanosomiasis control activities into primary health services in the democratic republic of the Congo: A qualitative study of stakeholder perceptions. Am J Trop Med Hyg 100: 899-906.

4. Bozdech V, Mason P (1992) The chemotherapy of human parasitic disease. University of Zimbabwe, Harare, 60-64.

5. Njiru ZK, Mikosza ASJ, Matovu E, Enyaru JCK, Ouma JO, et al. (2008) African trypanosomiasis: Sensitive and rapid detection of the sub-genus Trypanozoon by loop-mediated isothermal amplification (LAMP) of parasite DNA. Int $\mathrm{J}$ Parasitol 38: 589-599.

6. Kennedy PG (2013) Clinical features, diagnosis, and treatment of human African trypanosomiasis (sleeping sickness). Lancet Neurol 12: 186-194.

7. Truc $\mathrm{P}$, Lejon $\mathrm{V}$, Magnus $\mathrm{E}$, Jamonneau $\mathrm{V}$, Nangouma A, et al. (2002) Evaluation of the micro-CATT, CATT/ Trypanosoma brucei gambiense, and LATEX/T b gambiense methods for serodiagnosis and surveillance of human African trypanosomiasis in west and central Africa. Bull World Health Organ 80: 882-886.

8. Kuboki N, Inoue N, Sakurai T, Di Cello F, Grab DJ, et al. (2003) Loop-mediated isothermal amplification for detection of African Trypanosomes. J Clin Microbiol 41: 5517-5524.

9. Grab DJ, Nikolskaia OV, Courtioux B, Thekisoe OMM, Magez S, et al. (2019) Using detergent-enhanced LAMP for African trypanosome detection in human cerebrospinal fluid and implications for disease staging. PLoS Negl Trop Dis 13: e0007631.

10. Dama E, Camara O, Kaba D, Koffi M, Camara M, et al. (2019) Immune trypanolysis test as a promising bioassay to monitor the elimination of gambiense human African trypanosomiasis. Parasite 26: 68.

11. Koning HPD (2020) The drugs of sleeping sickness: Their mechanisms of action and resistance, and a brief history. Trop Med Infect Dis 5: 14.

12. WHO (2017) Report of the second WHO stakeholders meeting on rhodesiense human African trypanosomiasis, Geneva, 26-28 April 2017.

13. Gao JM, Qian ZY, Hide G, Lai DH, Lun ZR, et al. (2020) Human African trypanosomiasis: The current situation in endemic regions and the risks for non-endemic regions from imported cases. Parasitology 147: 922-931. 